\title{
Prospects for Early Discoveries in Final States with Dileptons and Jets: LRSM and Leptoquarks
}

\author{
Chaouki Boulahouache
}

University Of Pittsburgh, On Behalf of ATLAS Collaboration

\begin{abstract}
Studies based on fully simulated data samples containing two or more high $p_{T}$ leptons and jets have been performed in view of searching for BSM physics with a few $100 \mathrm{pb}^{-1}$ of early ATLAS [1] data, at $14 \mathrm{TeV} p p$ center-of-mass energy. We study the possibility of an early discovery of first and second generation leptoquark pairs, and of right-handed $W$ bosons and heavy neutrinos [2].
\end{abstract}

Keywords: Leptoquark, Left-Right symmetry, Heavy Right Handed $W$, Majorana neutrino PACS: 14.60.Cd, 14.60.Ef, 14.60.St, 14.70.Pw, 29.40.Vj, 29.85.Fj

\section{LEPTOQUARKS}

Leptoquarks (LQ) are bosons which directly couple to a lepton and a quark, therefore carrying baryon and lepton numbers, color, and fractional electric charge [3, 4], thus allowing lepton-quark transitions. They are naturally predicted in models which provide a possible explanation for the apparent symmetry between quarks and leptons, and can either be scalars or vectors. To evade experimental constraints from lepton number violation, flavorchanging neutral current, and proton decay, LQs are required to be family diagonal, allowing them to be light enough for being observable. At the LHC, LQs would be produced in pairs via the strong interaction (dominant) or in association with a lepton via leptoquark-quark-lepton coupling. Figure 1 shows the Feynman diagrams for LQ production processes at the LHC. We denote the branching fraction of a LQ to a charged lepton and a quark as $\beta$. For $\beta=1$, the highest lower limit [5] for 1st generation LQ (eq), $\mathrm{m}_{\mathrm{LQ}_{1}}>292 \mathrm{GeV}$, while for the 2 nd generation $\mathrm{LQ}(\mu \mathrm{q})$, the limit [6] is $316 \mathrm{GeV}$ (both based on $\left.1 \mathrm{fb}^{-1}\right)$. In this note, we present MC-based studies for 1st and 2nd generation scalar LQs generated at $\mathrm{m}=$ $400 \mathrm{GeV}$ using PYTHIA, where the NLO cross section is (2.24 \pm 0.376$)$ pb. Other mass points up to $800 \mathrm{GeV}$ have also been studied. We do not consider decays into neutrinos, and we do not select or identify events according to the quark flavor.

\section{LEFT-RIGHT SYMMETRIC MODELS}

In the standard model (SM), neutrinos are assumed to be massless, an assumption which was disproved by the discovery of neutrino oscillations. In Left-Right Symmetric models (LRSM), the introduction of new heavy right-handed Majorana neutrinos " $N_{\ell}$ " $(\ell=\mathrm{e}, \mu, \tau)$ induces tiny mass terms for the SM neutrinos via the Seesaw mechanism [7]. The $S U(3) \times S U(2)_{L} \times S U(2)_{R}$ gauge group of the LRSM also introduces new intermediate vector bosons $W_{R}$ 's and $Z^{\prime}$. The lower mass limits on $W_{R}$ bosons [8], established by the Tevatron experiments, are of $739 \mathrm{GeV}$, assuming the $W_{R}$ boson decays to both lepton pairs and quark pairs, and $768 \mathrm{GeV}$, assuming decays to quark pairs only. The new intermediate vector bosons can be produced at the LHC in the same processes as the SM's $W$ and $Z$ bosons. Their decays would be a source of the new Majorana neutrinos. A Feynman diagram for $W_{R}$ boson production and decay is shown in Fig. 2. Studies of the discovery potential for $W_{R}$ bosons were performed assuming a particular implementation [9] of the LRSM described in Ref. [10]. At LO, the production cross-sections $\sigma\left(p p \rightarrow W_{R} X\right)$ times branching fractions to the studied final states $W_{R} \rightarrow \ell N_{\ell} \rightarrow \ell \ell j j$ are $24.8 \mathrm{pb}$ for $\mathrm{M}\left(\mathrm{W}_{R}\right)=1800 \mathrm{GeV}, \mathrm{M}\left(N_{\ell}\right)=300 \mathrm{GeV}$ (referred to as LRSM_18_3), and $47 \mathrm{pb}$ for $\mathrm{M}\left(W_{R}\right)=1500$ $\mathrm{GeV}, \mathrm{M}\left(N_{\ell}\right)=500 \mathrm{GeV}$ (referred to as LRSM_15_5). The two leptons can be of same-sign and opposite sign, due to the Majorana nature of $N_{\ell}$.

\section{SELECTION CRITERIA \& RECONSTRUCTION}

The new resonances are relatively heavy objects, hence we require at least two high $p_{T}$ leptons and two high $p_{T}$ jets. Events must satisfy the three levels of the single lepton triggers with low thresholds. The overall efficiency of this trigger is high for the signal of interest. Signal lepton candidates are required to have $p_{T} \geq 20 \mathrm{GeV}$ and $|\eta| \leq 2.5$, where $\eta$ is the pseudorapidity. Furthermore, for electrons, we require that the energy clusters in the EM calorimeter and the matched track in the inner detector satisfy shower-shape and track-quality selection 


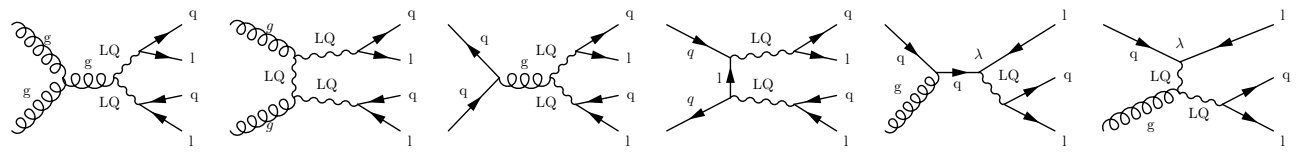

FIGURE 1. Leptoquark production and decay at the LHC.

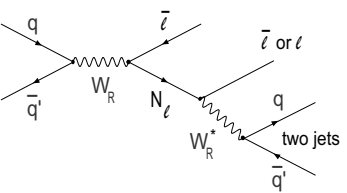

FIGURE 2. $W_{R}$ boson production and decay at the LHC.

criteria, respectively. Muon candidates are identified by matching tracks reconstructed in the inner detector with the tracks found in the muon spectrometer. They should also satisfy isolation requirements in the calorimeter. The two highest energy clusters $\left(p_{T} \geq 20 \mathrm{GeV}\right.$ and $|\eta| \leq 4.5$ ) that do not satisfy electron identification criteria are assumed to be the signal jets. A minimal angular separation of 0.1 rad between a jet and any electron candidate is imposed to reject electrons being misidentified as jets. A minimal preselection cut of $70 \mathrm{GeV}$ is also applied to the dilepton invariant mass to suppress the Drell-Yan background, while tighter analysis-specific cuts are applied in order to achieve the best sensitivities. The LQ invariant masses are reconstructed by combining any of the two high $p_{T}$ jets with any of the two high $p_{T}$ leptons, and keeping the combinations for which the difference between the two invariant masses is the smallest. Similarly, the $N_{\ell}$ neutrino invariant mass is reconstructed by combining the two highest $p_{T}$ jets with one of the two highest $p_{T}$ leptons, and keeping the combination which gives the smallest invariant mass. The $W_{R}$ boson invariant mass is reconstructed by combining the $N_{\ell}$ neutrino with the remaining high $p_{T}$ lepton. It is worth noting that for the dielectron channel, in the cases where $0.1<\Delta \mathrm{R}(\mathrm{e}$, jet $)<0.4$, only jets are used in the invariant mass reconstruction in order to avoid double counting.

\section{BACKGROUND STUDIES}

The most important sources of background considered in the presented analyses are: $t \bar{t}, Z / D Y+$ jets, dibosons $(W W, W Z$ and $Z Z)$ and multijet production processes where two jets are misidentified as electrons. Other potential background sources, such as single-top production, were also studied, but their contribution was found to be insignificant. To improve the signal to background ratio, selections based on the sum of the $p_{T}$ 's of the two
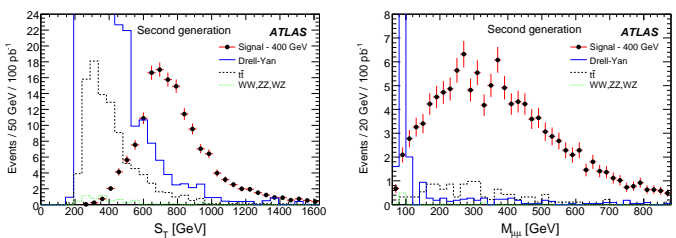

FIGURE 3. $S_{T}$ and $\mathrm{M}_{\mu \mu}$ distributions (LQ \& Background).
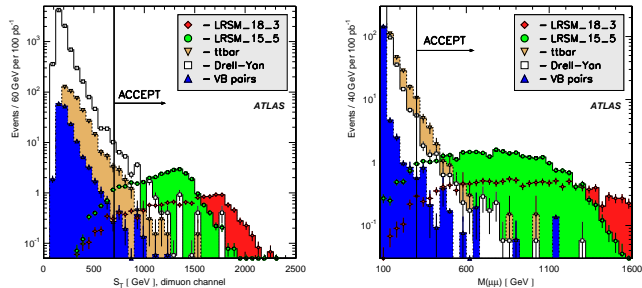

FIGURE 4. $S_{T}$ and $\mathrm{M}_{\mu \mu}$ distributions (LRSM \& Background).

leading leptons and the two leading jets $\left(\mathrm{S}_{\mathrm{T}}\right)$, and on the dilepton invariant mass, $\mathrm{M}(\ell \ell)$, were applied. In the LQ analysis, the background gets further reduced by requiring each of the $\mathrm{M}(\ell+\mathrm{jet})$ to be close to the tested LQ mass (dielectron channel) or the average mass to be consistent with the tested LQ mass (dimuon channel). Furthermore, we require that the two leptons be of opposite charge. Figure 3 (4) shows the comparison between LQ (LRSM) and the main SM backgrounds for the $\mathrm{S}_{\mathrm{T}}$ and $\mathrm{M}(\ell \ell)$ distributions in the muon channels. The cuts on $S_{T}$ and $\mathrm{M}(\ell \ell)$ are chosen to achieve $5 \sigma$ discovery with the smallest luminosity. The invariant mass distribution after applying all cuts is shown in Fig. 5 (6) for LQ (LRSM).
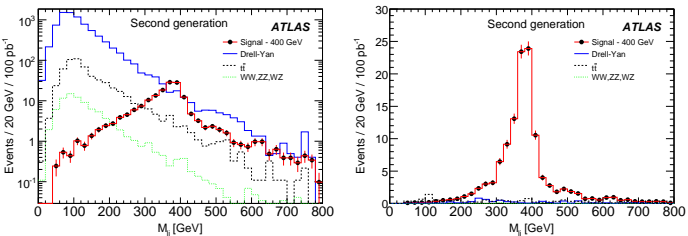

FIGURE 5. $\mu$-jet invariant mass for signal and background MC events before (left) and after (right) background suppression (normalized to $100 \mathrm{pb}^{-1}$ ) . 

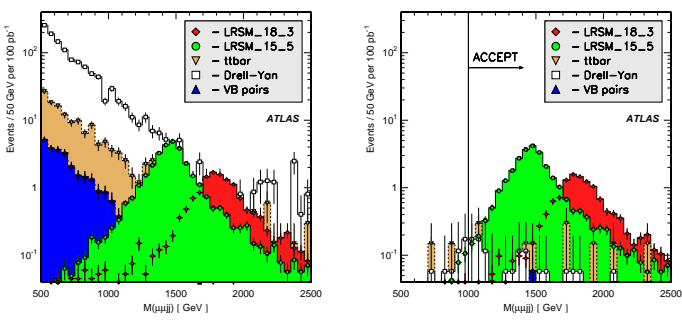

FIGURE 6. Dimuon-dijet invariant mass for signal and background MC events before (left) and after (right) background suppression (normalized to $100 \mathrm{pb}^{-1}$ ).

\section{SYSTEMATIC UNCERTAINTIES}

To estimate what will be the systematic uncertainties on LQ and LRSM analyses with early data $\left(100 \mathrm{pb}^{-1}\right)$, we considered contributions from the luminosity determination, lepton identification, trigger and reconstruction efficiencies, and lepton and jet energy scale and resolution. The systematic effects are dominated by the uncertainty in integrated pp luminosity $(20 \%)$, the jet energy scale (16\%-35\%), jet energy resolution (6\%-28\%), and the limited statistics of background MC samples (15\%$30 \%$ ). The overall relative systematic uncertainty on DY and $t \bar{t}$ backgrounds is expected to be approximately $45 \%$ and $40 \%$ in the dielectron and dimuon analyses, respectively. For the dielectron channels, the systematic uncertainty for multijet background will need to be understood with real data.

\section{SENSITIVITY \& DISCOVERY POTENTIAL}

The luminosities needed for a $5 \sigma$ discovery of 1 st and 2nd generation scalar LQ signals are shown in Fig. 7 for different values of $\beta^{2}$. The figure also shows a plot of the minimum values of $\beta^{2}$ that can be probed with $100 \mathrm{pb}^{-1}$ of ATLAS data as a function of LQ mass. The maximal LQ masses to which ATLAS is sensitive ( $\beta=$ 1) assuming $100 \mathrm{pb}^{-1}$ of data are expected to be $565 \mathrm{GeV}$ and $575 \mathrm{GeV}$ for 1 st and $2 \mathrm{nd}$ generation LQ respectively. For $W_{R}$ bosons, the significance of the signals and the minimal cross section times dilepton branching fraction necessary for a $5 \sigma$ discovery are plotted, in Fig. 8, as a function of the total integrated luminosity.

\section{CONCLUSIONS}

Studies of final states with two leptons and multiple jets have been discussed, considering both electrons and
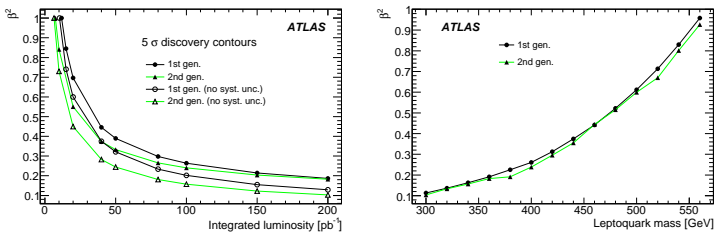

FIGURE 7. $5 \sigma$ discovery potential for scalar LQ $\left(\mathrm{m}_{\mathrm{LQ}}=\right.$ $400 \mathrm{GeV}$ ) vs. $\beta^{2}$ (left). Minimum $\beta^{2}$ vs. LQ mass for $5 \sigma$ discovery reach (background systematics included) (right).
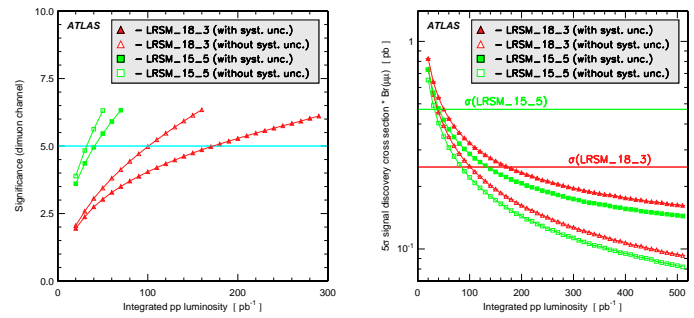

FIGURE 8. Signal significance vs. luminosity for $W_{R}$ bosons (left). Signal cross section $\times$ Dimuon branching fraction for a $5 \sigma$ discovery potential vs. luminosity (right).

muons. The early data discovery potential for BSM physics predicted by two prominent GUT-inspired models has been investigated. Both 1st and 2nd generation scalar LQ pair production could be discovered with less than $100 \mathrm{pb}^{-1}$ of integrated luminosity, provided that the mass of the LQ is smaller than $500 \mathrm{GeV}$ and $\beta=1$. Two LRSM mass points LRSM_18_3 and LRSM_15_5 for the $W_{R}$ bosons and Majorana neutrinos have been studied. The discovery of these new particles at these mass points would require integrated luminosities of 150 $\mathrm{pb}^{-1}$ and $40 \mathrm{pb}^{-1}$, respectively.

\section{REFERENCES}

1. G. Aad, et al., JINST 3 S08003 (2008).

2. ATLAS Collaboration, CERN-OPEN-2008-020 (2008, to appear.).

3. J. C. Pati, and A. Salam, Phys. Rev. D10, 275-289 (1974).

4. E. Eichten, K. D. Lane, and M. E. Peskin, Phys. Rev. Lett. 50, 811-814 (1983).

5. DO Conference Note 5644-CONF (2008).

6. V. M. Abazov, et al. (2008), arXiv: 0808.4023v1.

7. R. N. Mohapatra, and P. B. Pal, World Sci. Lect. Notes Phys. 60, 1-397 (1998).

8. V. M. Abazov, et al. (2008), arXiv:0803.3256.

9. A. Ferrari, et al., Phys. Rev. D62, 013001 (2000).

10. K. Huitu, J. Maalampi, A. Pietila, and M. Raidal, Nucl. Phys. B487, 27-42 (1997). 